\title{
Relação Hipsométrica para Pinus taeda L. em Diferentes Fases do Ciclo de Corte
}

\author{
Marcos Felipe Nicoletti ${ }^{1}$, Karine Souza ${ }^{1}$, Raul Silvestre ${ }^{1}$, \\ Morgana Cristina França ${ }^{1}$, Flávio Augusto Rolim²
}

${ }^{1}$ Departamento de Engenharia Florestal, Universidade do Estado de Santa Catarina - UDESC, Lages/SC, Brasil ${ }^{2}$ Departamento Florestal, Gerdau Florestal, Ponte Alta do Norte/SC, Brasil

\section{RESUMO}

O objetivo deste estudo foi avaliar as diferentes etapas do ciclo de corte, buscando uma relação hipsométrica adequada para estimar alturas dessas diferentes fases em um reflorestamento de Pinus taeda. Foram realizados cinco tratamentos, sendo o quinto a junção dos dados pertencentes aos demais tratamentos (modelo reduzido). A amostragem seguiu o método de área fixa, no qual o tamanho das parcelas e o número de árvores dominantes diferenciaram-se nos ciclos de corte. Definiu-se a localização das parcelas através de amostragem aleatória simples (oito parcelas por ciclo de corte). Foram determinadas as estatísticas de ajustes, coeficiente de determinação ajustado, erro-padrão e análise de resíduos. Efetuou-se o teste de identidade de Graybill para verificação da necessidade da utilização de diferentes modelos para distintas classes de idade. Os resultados comprovaram que existem diferenças significativas entre adotar-se um modelo específico para cada fase e utilizar um único melhor modelo para o povoamento total.

Palavras-chave: altura, reflorestamento, manejo florestal.

\section{Hipsometric Rate for Pinus taeda L. at Different Stages of the Cutting Cycle}

\begin{abstract}
This study aimed to evaluate the different phases of the cutting cycle, seeking a proper hypsometric rate to estimate the height of these different phases in a reforestation of Pinus taeda. The study consisted of five treatments, being the fifth the combination of the data belonging to the other treatments. The sampling followed the fixed area method where plots size and number of dominant trees varied with each cutting cycle. The plots location was defined by simple random sampling (eight portions by cutting cycle). We determined the statistical adjustments, adjusted coefficient of determination, standard error and residual analysis. We performed the Graybill identity test to assess the need to use different models for different age classes. Results showed that there are significant differences between adopting a specific model to each stage and use a single best model for the full settlement.
\end{abstract}

Keywords: height, reforestation, forest management. 


\section{INTRODUÇÃO}

Nos inventários florestais normalmente é medido o diâmetro de todas as árvores presentes nas parcelas, enquanto que comumente apenas a altura de alguns indivíduos é aferida. Por conseguinte, esses dados são processados com o objetivo de se estabelecer uma relação de regressão da altura em função do diâmetro, sendo que tal relação será utilizada para estimar a altura das demais árvores em função dos diâmetros (Machado et al., 1994). Dessa forma, esse procedimento acaba tornando mais econômica a atividade do inventário florestal, além do que responde bem no que se refere à precisão, quando analisada para uma única espécie (Finger, 1999; Soares et al., 2006).

Conforme Bartoszeck et al. (2004), a relação hipsométrica até mesmo em povoamentos de mesma idade varia com a qualidade do sítio e com a densidade. Essa relação altura e diâmetro fornece uma curva que pode descrever as condições dessa floresta: inclinação íngreme da curva aparece para povoamentos jovens, com fraco desenvolvimento ou ainda sítios bons, enquanto que inclinação achatada descreve um povoamento mais velho, momento em que o incremento corrente anual está caindo ou estado "clímax".

Os modelos matemáticos então entram em cena a fim de facilitar o processo do inventário florestal e Bartoszeck et al. (2004) descrevem que atualmente existem modelos modificados que incluem variáveis como idade, sítio, densidade e $\mathrm{h}_{\text {dom }}$. Porém, não basta selecionar qualquer modelo e estimar a altura das árvores que não foram mensuradas. Finger (1992) destaca que para determinar o modelo matemático que melhor descreve a relação hipsométrica de um povoamento qualquer deve-se testar vários modelos e então selecionar o que melhor se ajusta às condições existentes.

Dessa forma, o estudo objetivou avaliar se as diferentes etapas em que são conduzidas melhoram as estimativas em termos de precisão, a fim de que se obtenham melhores resultados, aliados a menores custo e tempo, propondo buscar uma relação hipsométrica ótima para estimar altura para as diferentes fases do ciclo de corte.

\section{MATERIAL E MÉTODOS}

\subsection{Caracterização da área}

A área experimental localiza-se no município de Ponte Alta do Norte, SC, com coordenadas geográficas $27^{\circ} 09^{\prime} 30^{\prime \prime}$ S e $50^{\circ} 27^{\prime} 52^{\prime \prime}$ W. A Fazenda Rio das Pedras, pertencente à empresa Florestal Rio Largo, abrange uma área total de 9.289,24 ha, dos quais $5.540,7$ ha compreendem florestas plantadas. O clima da região é temperado com estações bem definidas, mesotérmico úmido, com verão fresco e temperatura média de $15,5^{\circ} \mathrm{C}$ e apresenta altitude de $962 \mathrm{~m}$ acima do nível do mar. A precipitação média anual varia entre 1.500 a $1.700 \mathrm{~mm}$, enquanto que a precipitação máxima em 24 horas é de $140 \mathrm{~mm}$.

\subsection{Inventário florestal}

A Tabela 1 demonstra as fases do ciclo de corte em que foram realizadas as coletas de dados na Fazenda Rio das Pedras. Na escolha dos talhões para realização do presente estudo definiu-se que as áreas deveriam apresentar uma mesma qualidade de sítio, nesse caso, sítio médio, caracterizado pela empresa. Portanto, cada ciclo de corte foi assinalado por um tratamento

Tabela 1. Etapas do ciclo de corte estudadas e suas informações sobre idade, época de plantio e tamanho das parcelas para cada diferente ciclo de corte.

Table 1. Cutting cycle stages studied and their information on age, time of planting and plot size for each different cutting cycle.

\begin{tabular}{|ccccc|}
\hline Fase do ciclo de corte & Tratamento & Idade (anos) & Plantio & $\begin{array}{c}\text { Tamanho das } \\
\text { parcelas }\left(\mathbf{m}^{2}\right)\end{array}$ \\
\hline Antes do $1^{\circ}$ desbaste & 1 & 7 & jul/05 & $300(15 \times 20)$ \\
\hline Antes do $2^{\circ}$ desbaste & 2 & 8 & jun/04 & $450(15 \times 30)$ \\
\hline Antes do $4^{\circ}$ desbaste & 3 & 18 & out $/ 94$ & $750(15 \times 50)$ \\
\hline Antes do corte raso & 4 & 18 & abr $/ 94$ & $900(15 \times 60)$ \\
\hline Total de dados (Modelo reduzido) & 5 & - & - & - \\
\hline
\end{tabular}


diferenciado. No momento da coleta dos dados não havia uma área que atendesse a qualidade de sítio médio e ciclo de corte antes do terceiro desbaste, dessa maneira não foi possível analisar tal situação.

De acordo com o manejo de desbaste adotado pela empresa, houve um empecilho com a fase antes do corte raso, pois essa apresenta 18 anos, idade na qual teoricamente ainda não ocorreu o quarto desbaste, uma vez que esse é feito aos 19 anos.

O quinto tratamento consistiu em uma junção de dados, na qual as informações dendrométricas de todas as fases do ciclo de corte foram unidas.

O inventário florestal executado seguiu o método de área fixa, em que o tamanho das parcelas e o número de árvores dominantes variaram de acordo com cada ciclo de corte. Através do processo de amostragem aleatória simples, a localização de cada parcela ficou definida, sendo as parcelas alocadas em oito unidades para cada fase do ciclo de corte.

Todos os indivíduos tiveram sua circunferência à altura do peito mensurada por fita métrica. Em se tratando de altura, foi aferida a das 20 primeiras árvores, além das dominantes. A altura dominante é conceituada como a altura média das 100 árvores de maior diâmetro por hectare (Prodan, 1965; Assman, 1970).

\subsubsection{Relação hipsométrica}

a) Modelos hipsométricos

Selecionaram-se seis modelos hipsométricos (Tabela 2), a fim de que se pudesse escolher o melhor modelo para estimar a altura em cada fase do inventário florestal.

\section{b) Critérios de seleção dos modelos}

Sanquetta et al. (2009) prescrevem que a característica da melhor equação ajustada é avaliada por meio de parâmetros de comparação, a qual aponta o quanto as variáveis estão relacionadas e até mesmo qual o erro existente quando se está estimando parâmetros utilizando o modelo ajustado.

Para selecionar um modelo que, na média de todas as análises, obteve o melhor desempenho, adotou-se um ordenamento entre eles. Cada equação foi pontuada de 1 a 6, sendo que o menor valor corresponde ao melhor modelo, por conseguinte, o menor somatório foi considerado como o modelo mais adequado para estimar a altura. Para a seleção dos melhores modelos
Tabela 2. Modelos de relação hipsométrica ajustados para a espécie Pinus taeda (Equações 1-6).

Table 2. Hypsometric relation models adjusted for Pinus taeda (Equations 1-6).

\begin{tabular}{ccc} 
Equação & Autor & Modelo matemático \\
\hline 1 & Henriksen & $h=b_{0}+b_{1} \ln d$ \\
\hline 2 & Não referenciado & $\ln h=b_{0}+b_{1} d+b_{2} d^{2}$ \\
\hline 3 & Parabólico & $h=b_{0}+b_{1} d+b_{2} d^{2}$ \\
4 & Assman & $h=b_{0}+b_{1} \frac{1}{d}$ \\
\hline 5 & Stoffels & $\ln h=b_{0}+b_{1} \ln d$ \\
\hline 6 & $\begin{array}{c}\text { Azevedo et al. } \\
(1999)\end{array}$ & $\ln h=b_{0}+b_{1} \ln d+b_{2} \frac{1}{d}$ \\
\hline
\end{tabular}

$\ln =$ logaritmo neperiano; $\mathrm{h}=$ altura total $(\mathrm{m}) ; \mathrm{d}=$ diâmetro a 1,3 m do solo $(\mathrm{cm})$; b0, b1, b2 = coeficientes de regressão.

matemáticos foram levados em consideração os seguintes parâmetros estatísticos: Erro-padrão (Syx), Erro-padrão relativo (\%), Coeficiente de determinação ajustado $\left(\mathrm{R}^{2}\right.$ ajustado) e Índice de Schlaegel (I.A.), recalculados quando necessário, além da análise gráfica de resíduos.

$\mathrm{O}$ erro-padrão expressa o quanto em termos médios os valores observados variam em relação aos valores estimados. Portanto, valores menores expõem modelos melhores. O erro-padrão relativo, representa o erro-padrão em porcentagem, sendo obtido pela razão entre esse último e a média da variável dependente. De igual maneira, quanto menor for esse valor, melhor é o modelo. O coeficiente de determinação ajustado mede de 0 até 1 ou, ainda, de $0 \%$ a 100\% a explicação da variação da variável dependente pela variação da variável independente. Quanto maior for esse valor, mais satisfatório será o modelo.

Os valores obtidos pelas equações logaritmizadas foram corrigidos pelo fator de correção de Meyer. Segundo Machado et al. (2002), quando multiplicado pelos volumes estimados por equações logarítmicas, esse fator corrige a discrepância logarítmica. Para a comparação da eficiência entre modelos de natureza, forma e número de coeficientes diferentes, foram calculados o Índice de Ajuste de Schlaegel (IA) e o coeficiente de determinação ajustado ( $\left.\mathrm{R}^{2} \mathrm{aj}\right)$, conforme sugerido por Silva et al. (2008). 
De forma complementar, foi realizada a análise gráfica dos resíduos, que compõe um dos principais critérios de seleção de um modelo, pois permite verificar se o ajuste foi bom ao longo de toda linha de regressão, se não há tendenciosidade aparente e se há problemas de heterocedasticidade de variância. Em caso de empate entre modelos, esse critério é decisivo.

c) Validação dos modelos

Após a realização do ajuste da altura dos diferentes modelos, para os cinco tratamentos (quatro classes de idade e modelo reduzido), selecionou-se o modelo que melhor estimou a altura de acordo com os critérios estabelecidos. Para avaliação da necessidade de construção de uma equação para cada idade, foi aplicado o teste de identidade de modelos descrito por Graybill (1976). De acordo com Queiroz et al. (2008), esse teste rejeita ou aceita uma determinada hipótese por meio da redução da soma de quadrados da regressão e uma de suas principais características é permitir a avaliação do comportamento de uma ou mais variáveis em condições distintas.

\section{RESULTADOS E DISCUSSÃO}

A Tabela 3 apresenta os resultados referentes à análise estatística descritiva da estrutura diamétrica do plantio de Pinus taeda nos diferentes ciclos de corte.

Pode-se afirmar que com o aumento do número de desbastes existe um aumento do diâmetro (Tabela 3), com destaque para a pequena diferença entre a fase antes do quarto desbaste e a fase antes do corte raso. Essa pequena diferença entre a variação dos diâmetros pode ser explicada pela antecipação do quarto desbaste da última situação.

Os resultados referentes aos ajustes dos seis modelos de relação hipsométrica, para os cinco diferentes tratamentos, estão apresentados nas Tabelas 4, 5, 6, 7 e 8 .

As equações hipsométricas apresentaram coeficiente de determinação ajustados semelhante, salvo uma maior variação no tratamento 2, onde o modelo de Assman obteve pior resultado. Nos tratamentos 1 e 4, o modelo Stoffels obteve valores menores de $\mathrm{R}_{\text {aj }}^{2}$, enquanto que para os tratamentos 2 e 5 o modelo de Assman foi o que apresentou pior resultado, levando-se em consideração a estatística do $\mathrm{R}_{\text {aj. }}^{2} \mathrm{O}$ modelo de Azevedo foi pior apenas para o tratamento 3 .

No entanto, não se recomenda utilizar unicamente o coeficiente de determinação para avaliar o ajuste de um modelo. O Índice de Schlaegel (I.A.), assim como o $\mathrm{R}_{\text {aj }}^{2}$, considera os modelos com maiores valores como os que melhor se ajustam. Para o tratamento 1, o I.A. variou de 0,4303 até 0,4520; no tratamento 2 variou entre 0,3619 e 0,4232; no tratamento 3 variou de 0,1810 até 0,1929; no tratamento 4 variou entre 0,2543 e 0,2663; e, finalmente, no tratamento 5 variou de 0,8148 a 0,8722 . Logo, analisando esses dados, percebe-se que o modelo 2 conseguiu melhor desempenho para os tratamentos 2 e 5, enquanto que o modelo parabólico foi melhor para o restante dos tratamentos.

O erro-padrão da estimativa $\left(\mathrm{S}_{\mathrm{yx}} \%\right)$ aliado à análise gráfica de resíduos constituem os mais importantes meios para seleção de um modelo, portanto são fatores decisivos no momento da escolha.

Tabela 3. Análise estatística descritiva do diâmetro a 1,3 $\mathrm{m}$ do solo para a espécie Pinus taeda nos diferentes ciclos de corte e idades de um reflorestamento em Santa Catarina.

Table 3. Descriptive statistical analysis of the diameter at $1.3 \mathrm{~m}$ above the ground for the species Pinus taeda in different cutting cycles and ages of a reforestation in Santa Catarina.

\begin{tabular}{|c|c|c|c|c|c|c|}
\hline Ciclo de corte & Idade (anos) & Mínimo $(\mathrm{cm})$ & Máximo (cm) & Média $(\mathrm{cm})$ & $\begin{array}{c}\text { Desvio- } \\
\text { padrão }(\mathrm{cm})\end{array}$ & $\begin{array}{c}\text { Coeficiente } \\
\text { de variação } \\
(\%)\end{array}$ \\
\hline $\begin{array}{l}\text { Antes do } 1^{\circ} \\
\text { desbaste }\end{array}$ & 7 & 8,9 & 25,2 & 18,1 & 2,7 & 14,89 \\
\hline $\begin{array}{l}\text { Antes do } 2^{\circ} \\
\text { desbaste }\end{array}$ & 8 & 12,9 & 32,2 & 21,4 & 3 & 14,01 \\
\hline $\begin{array}{l}\text { Antes do } 4^{\circ} \\
\text { desbaste }\end{array}$ & 18 & 23,3 & 50,9 & 33,2 & 4,8 & 14,53 \\
\hline $\begin{array}{l}\text { Antes do corte } \\
\text { raso }\end{array}$ & 18 & 24,9 & 49,6 & 33,8 & 4,3 & 12,77 \\
\hline
\end{tabular}


Tabela 4. Parâmetros estatísticos dos modelos de relação hipsométrica ajustados para o tratamento1 referente ao ciclo de corte antes do primeiro desbaste para a espécie Pinus taeda em Santa Catarina.

Table 4. Statistical parameters of the models hypsometric relation adjusted for treatment 1 for the cutting cycle before the $1^{\text {st }}$ thinning, for the species Pinus taeda in Santa Catarina.

\begin{tabular}{|c|c|c|c|c|c|c|}
\hline \multirow{2}{*}{$\mathbf{N}^{\circ}$} & \multicolumn{3}{|c|}{ Coeficientes da Regressão } & \multirow[b]{2}{*}{$\mathrm{S}_{\mathrm{yx}} \%$} & \multirow[b]{2}{*}{$\mathbf{R}_{\text {aj }}^{2}$} & \multirow[b]{2}{*}{ IA } \\
\hline & $b_{0}$ & $b_{1}$ & $b_{2}$ & & & \\
\hline 1 & $-3,48318^{\star \star}$ & $4,97377^{\star \star}$ & - & 8,08 & 0,42695 & 0,4303 \\
\hline 2 & $1,25283^{* *}$ & $0,09972^{\star *}$ & $-0,00202^{* *}$ & 8,06 & 0,45201 & 0,45201 \\
\hline 3 & $-0,03819^{\mathrm{ns}}$ & $0,94069^{\star *}$ & $-0,01828^{*}$ & 8,06 & 0,42981 & 0,43648 \\
\hline 4 & $15,75736^{\star *}$ & $-86,50911^{\star *}$ & - & 8,05 & 0,4313 & 0,43462 \\
\hline 5 & $1,01801^{\star \star}$ & $0,47199^{\star *}$ & - & 8,11 & 0,44487 & 0,44487 \\
\hline 6 & $3,74993^{*}$ & $-0,23434^{\text {ns }}$ & $-12,27671^{\mathrm{ns}}$ & 8,07 & 0,45032 & 0,45032 \\
\hline
\end{tabular}

${ }^{* *}$ significativo ao nível de $1 \%$ de significância. ${ }^{*}$ significativo ao nível de $5 \%$ de significância. ${ }^{\text {ns }}$ não significativo ao nível de $5 \%$ de significância.

Tabela 5. Parâmetros estatísticos dos modelos de relação hipsométrica ajustados para o tratamento 2 referente ao ciclo de corte antes do segundo desbaste para a espécie Pinus taeda em Santa Catarina.

Table 5. Statistical parameters of the models hypsometric relation adjusted for treatment 2 for the cutting cycle before the $2^{\text {nd }}$ thinning, for the species Pinus taeda in Santa Catarina.

\begin{tabular}{|c|c|c|c|c|c|c|}
\hline \multirow{2}{*}{$\mathbf{N}^{\circ}$} & \multicolumn{3}{|c|}{ Coeficientes da Regressão } & \multirow{2}{*}{$S_{y x} \%$} & \multirow{2}{*}{$\mathbf{R}_{\text {aj }}^{2}$} & \multirow{2}{*}{ IA } \\
\hline & $b_{0}$ & $b_{1}$ & $b_{2}$ & & & \\
\hline 1 & $0,10768^{\mathrm{ns}}$ & $4,43733^{\star *}$ & - & 5,931 & 0,38729 & 0,39077 \\
\hline 2 & $2,53385^{\star *}$ & $-0,00756^{\mathrm{ns}}$ & $0,00051^{\mathrm{ns}}$ & 5,792 & 0,40744 & 0,40744 \\
\hline 3 & $13,16182^{\star *}$ & $-0,15879^{\text {ns }}$ & $0,00831^{\star}$ & 5,787 & 0,41661 & 0,42324 \\
\hline 4 & $17,920^{* *}$ & $-89,16754^{* *}$ & - & 6,07 & 0,35831 & 0,36195 \\
\hline 5 & $1,63392^{\star *}$ & $0,32015^{\star \star}$ & - & 5,896 & 0,38407 & 0,38407 \\
\hline 6 & $-1,39836^{\mathrm{ns}}$ & $1,06767^{\star *}$ & $15,70443^{* *}$ & 5,788 & 0,40706 & 0,40706 \\
\hline
\end{tabular}

${ }^{*}$ significativo ao nível de $1 \%$ de significância. ${ }^{*}$ significativo ao nível de $5 \%$ de significância. ${ }^{\text {ns }}$ não significativo ao nível de $5 \%$ de significância.

Tabela 6. Parâmetros estatísticos dos modelos de relação hipsométrica ajustados para o tratamento 3 referente ao ciclo de corte antes do quarto desbaste para a espécie Pinus taeda em Santa Catarina.

Table 6. Statistical parameters of the models hypsometric relation adjusted for treatment 3 for the cutting cycle before the $4^{\text {th }}$ thinning, for the species Pinus taeda in Santa Catarina.

\begin{tabular}{|c|c|c|c|c|c|c|}
\hline \multirow{2}{*}{$\mathbf{N}^{\mathbf{o}}$} & \multicolumn{3}{|c|}{ Coeficientes da Regressão } & \multirow{2}{*}{$S_{y x} \%$} & \multirow{2}{*}{$\mathbf{R}_{\text {aj }}^{2}$} & \multirow{2}{*}{ IA } \\
\hline & $b_{0}$ & $b_{1}$ & $b_{2}$ & & & \\
\hline 1 & $5,67150^{*}$ & $4,36803^{* *}$ & - & 6,209 & 0,18761 & 0,19195 \\
\hline 2 & $2,66606^{* *}$ & $0,01612^{\mathrm{ns}}$ & $-0,00014^{\mathrm{ns}}$ & 6,221 & 0,18201 & 0,18201 \\
\hline 3 & $13,29359^{* *}$ & $0,32669^{\mathrm{ns}}$ & $-0,00286^{\mathrm{ns}}$ & 6,222 & 0,1842 & 0,19292 \\
\hline 4 & $25,41750^{* *}$ & $-146,11981^{* *}$ & - & 6,212 & 0,18687 & 0,19122 \\
\hline 5 & $2,31612^{\star *}$ & $0,20689^{* *}$ & - & 6,210 & 0,18521 & 0,18521 \\
\hline 6 & $2,64433^{\text {ns }}$ & $-0,13438^{\text {ns }}$ & $-2,43959^{\text {ns }}$ & 6,226 & 0,181 & 0,18099 \\
\hline
\end{tabular}

Analisando esse resultado, conclui-se que o melhor modelo para os tratamentos 1 e 5 é o modelo 2; para o tratamento 2, o modelo que se sobressai é o parabólico; para o tratamento 3, o modelo de Henriksen obteve menor somatório; e, por fim, para o tratamento $4 \mathrm{o}$ melhor modelo foi o de Assman.

Todavia, a avaliação final levou em consideração ainda a análise do gráfico de resíduo, visto que através dele pode-se observar modelos que apresentem tendenciosidades ao longo da linha média.

Para o conjunto de dados abordado no presente estudo ressalta-se que a análise gráfica dos resíduos não se mostrou critério esclarecedor de seleção, pois todos os modelos apresentaram tendência similar, evidenciando ausência de tendenciosidade ao longo de toda linha de regressão (Figura 1). Dessa forma, apesar de não existir 
Tabela 7. Parâmetros estatísticos dos modelos de relação hipsométrica ajustados para o tratamento 4 referente ao ciclo de corte antes do corte raso para a espécie Pinus taeda em Santa Catarina.

Table 7. Statistical parameters of the models hypsometric relation adjusted for treatment 4 for the cutting cycle before the cut, for the species Pinus taeda in Santa Catarina.

\begin{tabular}{|c|c|c|c|c|c|c|}
\hline \multirow{2}{*}{$\mathbf{N}^{\circ}$} & \multicolumn{3}{|c|}{ Coeficientes da Regressão } & \multirow{2}{*}{$S_{y x} \%$} & \multirow{2}{*}{$\mathbf{R}_{\text {aj }}^{2}$} & \multirow{2}{*}{ IA } \\
\hline & $b_{0}$ & $b_{1}$ & $b_{2}$ & & & \\
\hline 1 & $-0,60631^{\mathrm{ns}}$ & $6,28954^{\star *}$ & - & 6,321 & 0,25464 & 0,25867 \\
\hline 2 & $2,33847^{\star *}$ & $0,03389^{* *}$ & $-0,00036^{*}$ & 6,305 & 0,25917 & 0,25917 \\
\hline 3 & $6,30657^{\mathrm{ns}}$ & $0,70342^{\star *}$ & $-0,00741^{*}$ & 6,305 & 0,25831 & 0,26633 \\
\hline 4 & $27,99279^{* *}$ & $-216,54051^{* *}$ & - & 6,3 & 0,25956 & 0,26356 \\
\hline 5 & $2,03927^{\star *}$ & $0,29195^{\star *}$ & - & 6,33 & 0,25432 & 0,25432 \\
\hline 6 & $4,76012^{*}$ & $-0,30722^{\text {ns }}$ & $-20,50605^{\text {ns }}$ & 6,31 & 0,25773 & 0,25773 \\
\hline
\end{tabular}

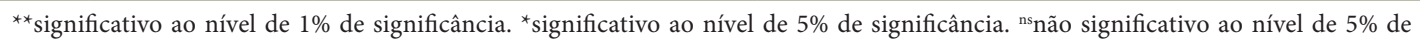
significância.

Tabela 8. Parâmetros estatísticos dos modelos de relação hipsométrica ajustados para o tratamento 5 referente à junção dos dados de todos os ciclos de corte para a espécie Pinus taeda em Santa Catarina.

Table 8. Statistical parameters of the models hypsometric relation adjusted for treatment related to junction 5 of the data of all cutting cycles for the species Pinus taeda in Santa Catarina.

\begin{tabular}{|c|c|c|c|c|c|c|}
\hline \multirow{2}{*}{$\mathbf{N}^{\circ}$} & \multicolumn{3}{|c|}{ Coeficientes da Regressão } & \multirow{2}{*}{$S_{y x} \%$} & \multirow{2}{*}{$\mathbf{R}_{\text {aj }}^{2}$} & \multirow{2}{*}{ IA } \\
\hline & $b_{0}$ & $b_{1}$ & $b_{2}$ & & & \\
\hline 1 & $-29,29359^{\star *}$ & $14,16537^{\star *}$ & - & 11,024 & 0,84631 & 0,84652 \\
\hline 2 & $1,08913^{\star *}$ & $0,09411^{\star *}$ & $-0,00108^{\star *}$ & 10,082 & 0,87225 & 0,87225 \\
\hline 3 & $-7,22716^{\star *}$ & $1,27384^{* *}$ & $-0,01313^{\star *}$ & 10,546 & 0,85934 & 0,85973 \\
\hline 4 & $30,46446^{* *}$ & $-336,64981^{* *}$ & - & 12,109 & 0,81458 & 0,81483 \\
\hline 5 & $-0,12691^{\star *}$ & $0,89257^{\star *}$ & - & 11,619 & 0,85334 & 0,85334 \\
\hline 6 & $0,62748^{\text {ns }}$ & $0,71502^{* *}$ & $-4,35369^{*}$ & 11,359 & 0,85398 & 0,85398 \\
\hline
\end{tabular}

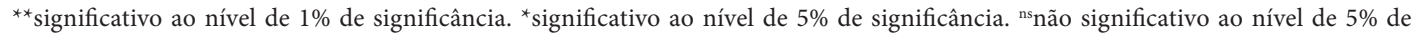
significância.

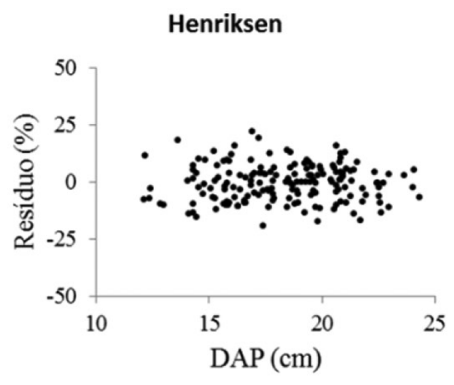

Assman

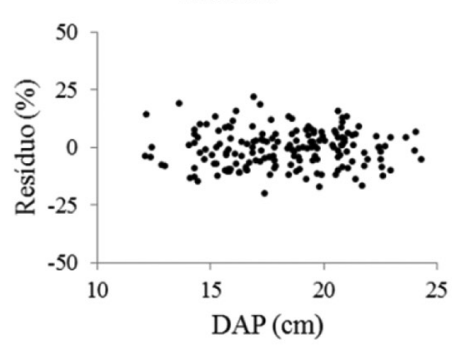

Modelo 2

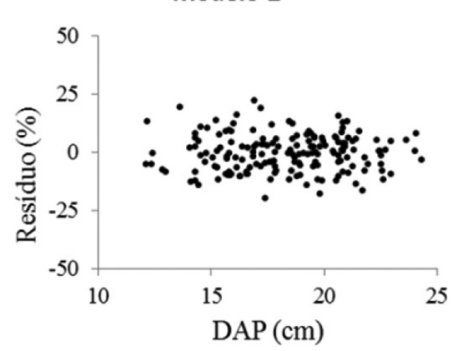

Stoffels

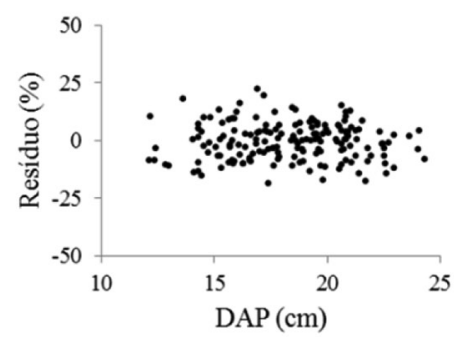

Parabólico

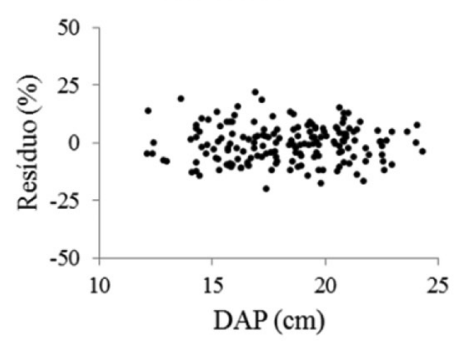

Azevedo

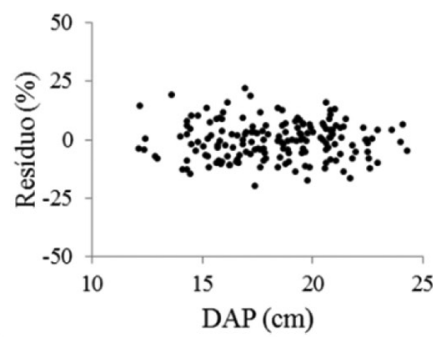

Figura 1. Relações hipsométricas de Pinus taeda, tratamento 1, em um reflorestamento localizado em Santa Catarina. Figure 1. Hypsometric relations of Pinus taeda, treatment 1, in a reforestation located in Santa Catarina. 
diferenças discrepantes entre as estatísticas de ajuste e seleção, o modelo 2 pode ser o mais indicado para estimativas hipsométricas no tratamento 1.

Assim como no tratamento 1, a distribuição gráfica dos resíduos (Figura 2) mostrou ausência de tendenciosidade aparente, com resíduos distribuídos uniformemente ao longo da linha de regressão. Dessa forma, o modelo que melhor estima a altura para o tratamento 2 é o parabólico.

Foi observado, no tratamento 3 (Figura 3), que a distribuição gráfica dos resíduos mostrou-se semelhante para as seis equações selecionadas, porém com destaque

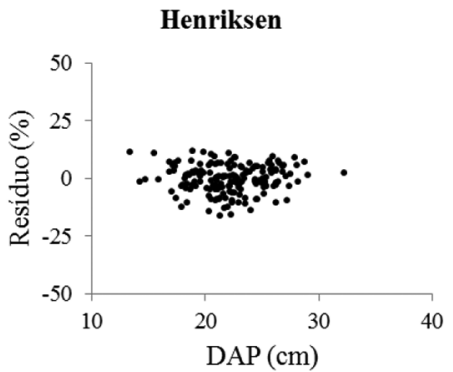

Assman

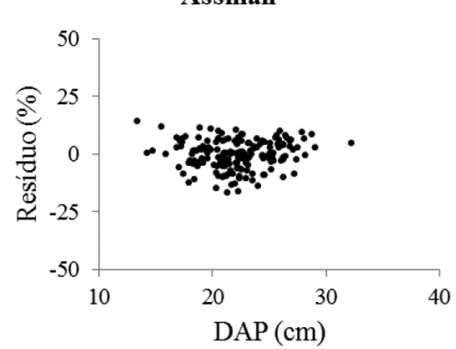

Modelo 2

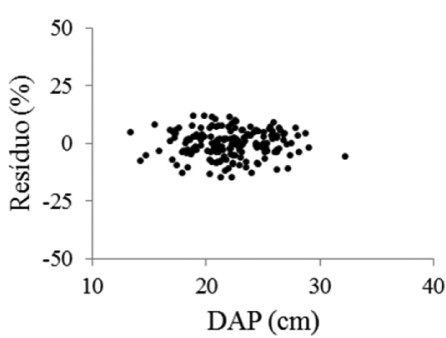

Stoffels

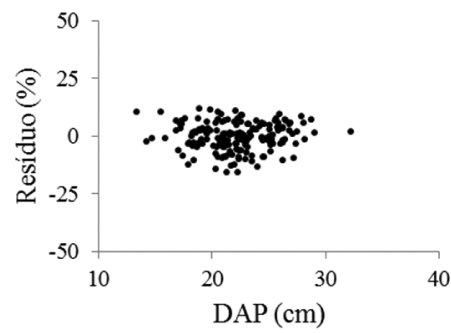

Parabólico

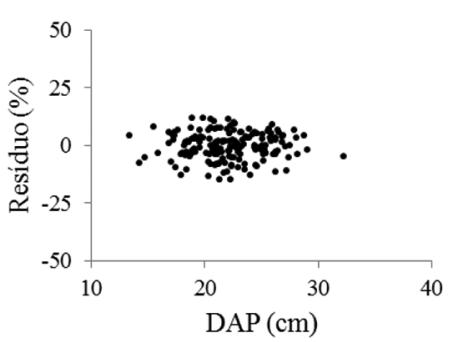

Azevedo

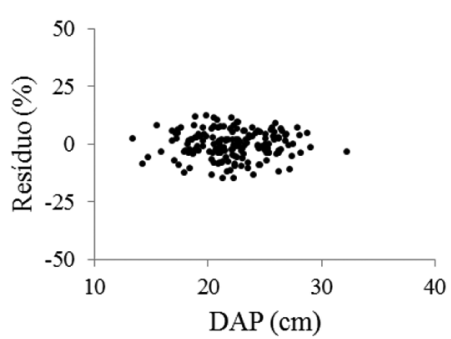

Figura 2. Relações hipsométricas de Pinus taeda, tratamento 2, em um reflorestamento localizado em Santa Catarina. Figure 2. Hypsometric relations of Pinus taeda, treatment 2, in a reforestation located in Santa Catarina.

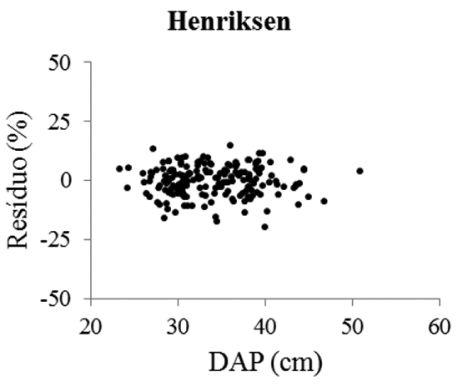

Assman

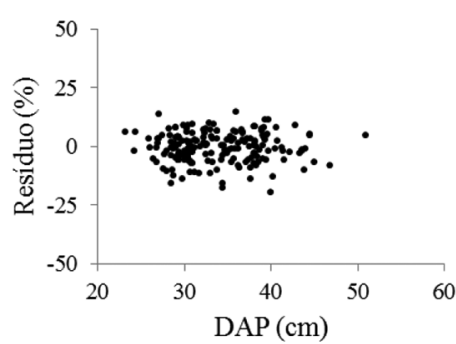

Modelo 2

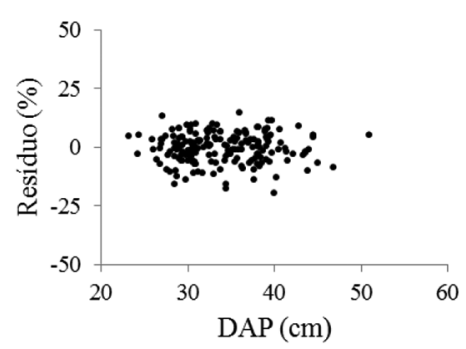

Stoffels

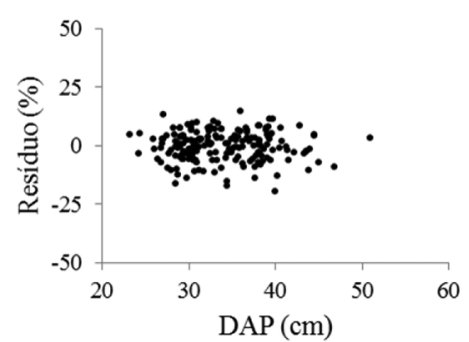

Parabólico

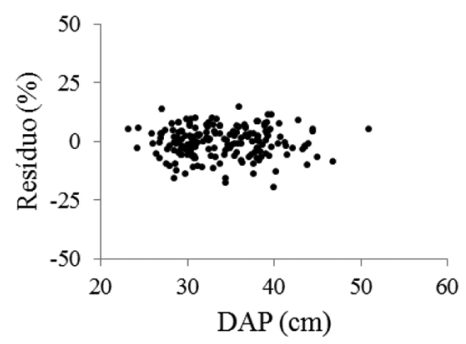

Azevedo

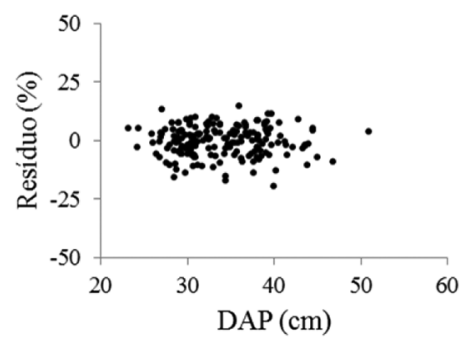

Figura 3. Relações hipsométricas de Pinus taeda, tratamento 3, em um reflorestamento localizado em Santa Catarina. Figure 3. Hypsometric relations of Pinus taeda, treatment 3, in a reforestation located in Santa Catarina. 
para melhor resíduo no modelo de Assman, e como os resultados estatísticos obtiveram pouca variação, o modelo que melhor se ajusta, para esse caso, é o de Assman.

Assim como ocorreu nos tratamentos 1 e 3, o tratamento 4 não demonstrou uma não tendenciosidade em toda a linha de regressão para todos os modelos testados (Figura 4) e, da mesma forma, optou-se por selecionar o modelo que obteve melhores parâmetros estatísticos, que foi o modelo de Assman.

O tratamento 5, correspondente ao modelo reduzido do teste de identidade de Graybill, apresentou distribuição heterocedástica, evidenciando problemas no ajuste de apenas um modelo global para todo o conjunto de dados (Figura 5). Por esse motivo realizou-se o

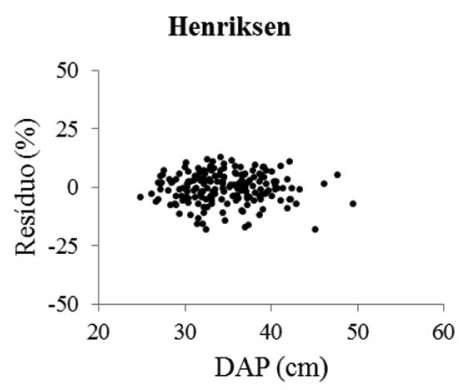

Assman

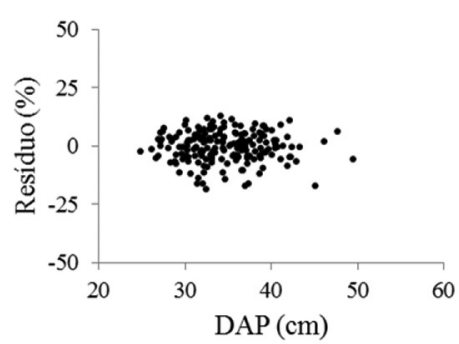

Modelo 2

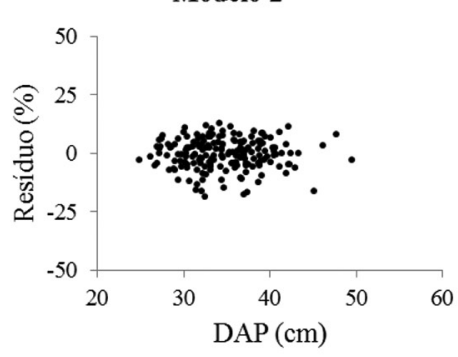

Stoffels

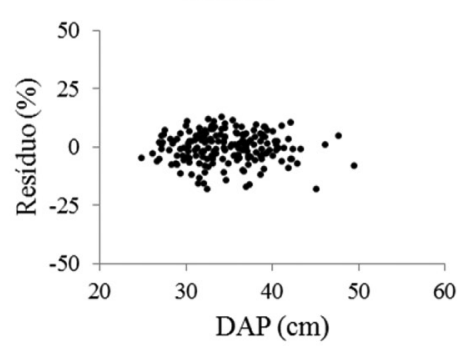

Parabólico

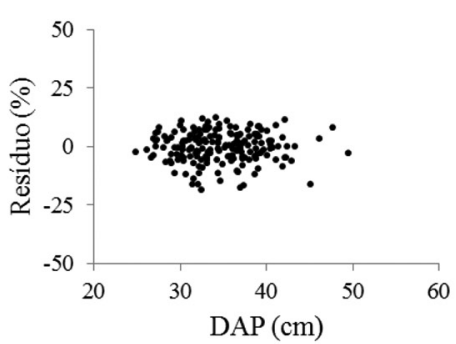

Azevedo

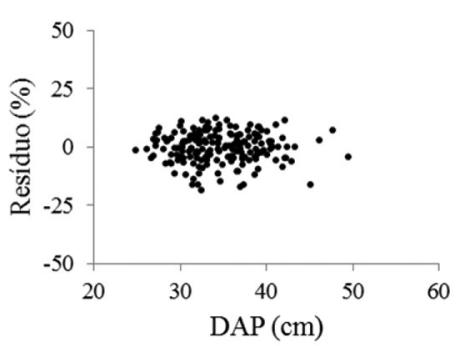

Figura 4. Relações hipsométricas de Pinus taeda, tratamento 4, em um reflorestamento localizado em Santa Catarina. Figure 4. Hypsometric relations of Pinus taeda, treatment 4, in a reforestation located in Santa Catarina.

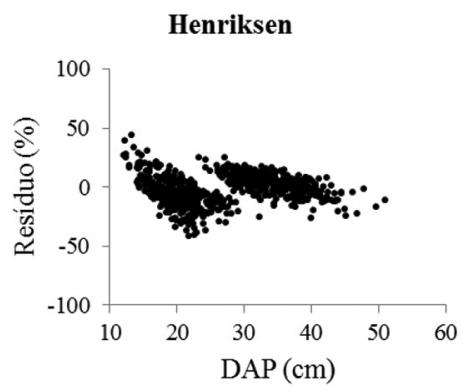

Assman

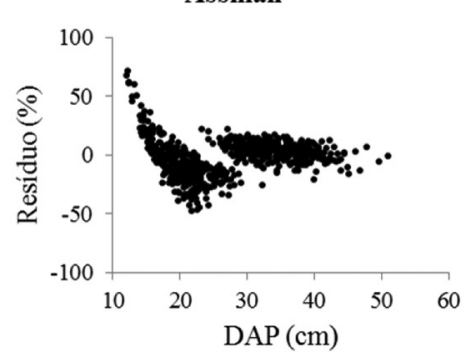

Modelo 2

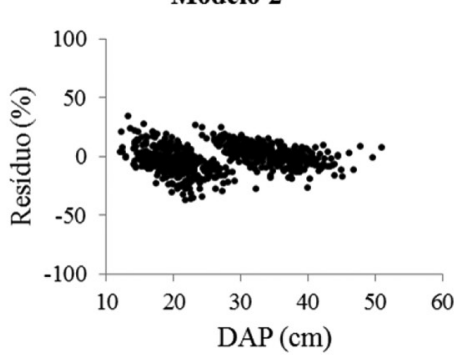

Stoffels

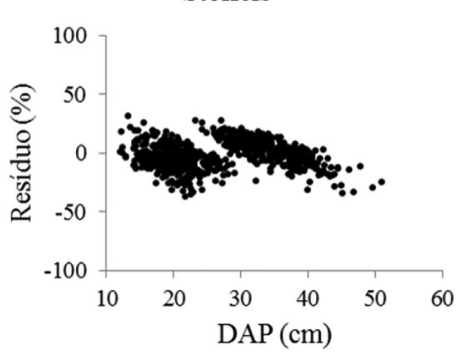

Parabólico

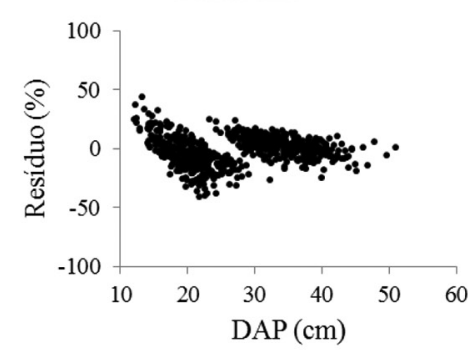

Azevedo

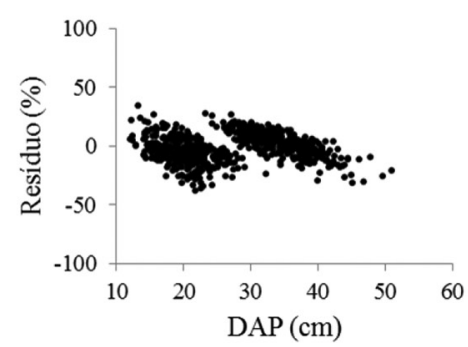

Figura 5. Relações hipsométricas de Pinus taeda, tratamento 5, em um reflorestamento localizado em Santa Catarina. Figure 5. Hypsometric relations of Pinus taeda, treatment 5, in a reforestation located in Santa Catarina. 
teste de identidade de Graybill para comprovação da necessidade de equações diferenciadas para cada tratamento. Cabe ressaltar que, devido à inexistência de uma fase de desbaste, os pontos no gráfico nesse tratamento, apresentam uma descontinuidade ao longo do eixo $\mathrm{x}$, uma vez que não foi encontrado nenhum diâmetro nesse intervalo.
O resultado do teste de Graybill para a hipótese de as equações serem idênticas está na Tabela 9 . O teste se apresentou significativo ao nível de $1 \%$ de probabilidade para todos os modelos ajustados em cada classe de idade, rejeitando-se a hipótese $\mathrm{H}_{0}$. Esse resultado indica que as equações diferiram significativamente entre si, não sendo recomendada a utilização do modelo

Tabela 9. Análise de variância para o teste de identidade de Graybill dos modelos hipsométricos testados nos tratamentos. Table 9. Analysis of variance for Graybill identity test of thehypsometric models in the treatments tested.

\begin{tabular}{|c|c|c|c|c|}
\hline \multicolumn{5}{|c|}{ Modelo 1} \\
\hline Fontes de variação & GL & SQ & QM & $\mathbf{F}$ \\
\hline Modelo completo & 8 & 224949,86 & & \multirow{5}{*}{212,56} \\
\hline Modelo reduzido & 2 & 223324,21 & & \\
\hline Redução & 6 & 1625,65 & 270,94 & \\
\hline Resíduo & 715 & 911,39 & 1,27 & \\
\hline Total & 723 & 225861,25 & & \\
\hline \multicolumn{5}{|c|}{ Modelo 2} \\
\hline Fontes de variação & GL & SQ & QM & F \\
\hline Modelo completo & 12 & 5694,19 & & \multirow{5}{*}{129,88} \\
\hline Modelo reduzido & 3 & 5689,03 & & \\
\hline Redução & 9 & 5,16 & 0,57 & \\
\hline Resíduo & 711 & 3,14 & 0,004 & \\
\hline Total & & 5697,32 & & \\
\hline \multicolumn{5}{|c|}{ Modelo 3} \\
\hline Fontes de variação & GL & SQ & QM & $\mathbf{F}$ \\
\hline Modelo completo & 12 & 224961,49 & & \multirow{5}{*}{124,58} \\
\hline Modelo reduzido & 3 & 223542,57 & & \\
\hline Redução & 9 & 1418,93 & 157,66 & \\
\hline Resíduo & 711 & 899,76 & 1,27 & \\
\hline Total & 723 & 225861,25 & & \\
\hline \multicolumn{5}{|l|}{ Modelo 4} \\
\hline Fontes de variação & GL & SQ & QM & $\mathrm{F}$ \\
\hline Modelo completo & 8 & 224947,30 & & \\
\hline Modelo reduzido & 2 & 222800,47 & & \\
\hline Redução & 6 & 2146,84 & 357,81 & 279,92 \\
\hline Resíduo & 715 & 913,95 & 1,28 & \\
\hline Total & 723 & 225861,25 & & \\
\hline \multicolumn{5}{|c|}{ Modelo 5} \\
\hline Fontes de variação & GL & SQ & $\mathbf{Q M}$ & $\mathbf{F}$ \\
\hline Modelo completo & 8 & 5694,13 & & \\
\hline Modelo reduzido & 2 & 5687,79 & & \\
\hline Redução & 6 & 6,34 & 1,06 & 236,53 \\
\hline Resíduo & 715 & 3,19 & 0,004 & \\
\hline Total & 723 & 5697,32 & & \\
\hline \multicolumn{5}{|c|}{ Modelo 6} \\
\hline Fontes de variação & GL & SQ & QM & $\mathbf{F}$ \\
\hline Modelo completo & 12 & 5694,18 & & \\
\hline Modelo reduzido & 3 & 5687,84 & & \\
\hline Redução & 9 & 6,34 & 0,70 & 161,99 \\
\hline Resíduo & 711 & 3,14 & 0,004 & \\
\hline Total & 723 & 5697,32 & & \\
\hline
\end{tabular}


reduzido para representar o conjunto de dados e a população abordada.

\section{CONCLUSÃO}

A relação hipsométrica para os povoamentos estudados se apresentou, de forma geral, como uma relação biológica fraca, evidenciada pelos reduzidos valores de coeficientes de determinação ajustados ( $\mathrm{R}^{2}$ aj.). No entanto, os erros-padrão das estimativas podem ser considerados satisfatórios.

Quanto ao teste de identidade de Graybill, foi possível concluir que os diferentes tratamentos exigem utilização de equações específicas e que o modelo global generalista representado pelo modelo reduzido não se mostra adequado para tais estimativas.

\section{STATUS DA SUBMISSÃO}

Recebido: 12 jul., 2013

Aceito: 13 dez., 2015

\section{AUTOR(ES) PARA CORRESPONDÊNCIA}

\section{Marcos Felipe Nicoletti}

Departamento de Engenharia Florestal, Universidade do Estado de Santa Catarina - UDESC, Av. Luiz de Camões, 2090, Conta Dinheiro, CEP 88520-000, Lages, SC, Brasil e-mail: marcos.nicoletti@udesc.br

\section{REFERÊNCIAS}

Assman E. The principles of forest yield study. Oxford: Pergamon Press; 1970. 506 p.

Azevedo CP, Muroya K, Garcia LC, Lima RMB, Moura JB, Neves EJM. Relação hipsométrica para quatro espécies florestais em plantio homogêneo e em diferentes idades na Amazônia Ocidental. Boletim de Pesquisa Florestal 1999; (39): 5-29.

Bartoszeck ACPS, Machado SA, Figueiredo A Fo, Oliveira EB. Dinâmica da relação hipsométrica em função da idade, do sítio e da densidade inicial de povoamentos de bracatinga da região metropolitana de Curitiba, PR. Revista Árvore 2004; 28(4): 517-533.

Finger CAG. Fundamentos da biometria florestal. Santa Maria: UFSM/CEPEF/FATEC; 1992. 269 p.

Finger CAG. Determinação do peso do desbaste para florestas de Eucalyptus grandis HILL ex MAIDEN, com base no espaçamento relativo. Ciência Florestal 1999; 9(1): 79-87.

Graybill FA. Theory and application of linear model. Pacific Grove: Belmont Duxbury; 1976. 704 p.

Machado SA, Bailey RL, Basso SF, Bevilacqua VG Jr. Análise do comportamento da relação hipsométrica com respeito à idade para plantações de Pinus elliotti no estado do Paraná. Cerne 1994; 1(1): 5-12.

Machado SA, Conceição MB, Figueiredo DJ. Modelagem do volume individual para diferentes idades e regimes de desbaste em plantações de Pinus oocarpa. Revista Ciências Exatas e Naturais 2002; 4(2): 41-50.

Prodan M. Holzmesslehre. Frankfurt-am Main: J. D. Sauerländer's Verlag; 1965. 644 p.

Queiroz D, Machado SA, Figueiredo A Jr, Arce JE, Koehler HS. Identidade de modelos em funções de afilamento para Mimosa scabrella Bentham em povoamentos nativos da região metropolitana de Curitiba/PR. Floresta 2008; 38(2): 339-349. http://dx.doi.org/10.5380/rf.v38i2.11629.

Sanquetta CR, Watzlawick LF, Côrte PD, Fernandes LAV. Inventários florestais: planejamento e execução. Curitiba: Multi-Graphic Gráfica e Editora; 2009. 319 p.

Silva GF, Leite VR, Curto RA, Mora R, Martins LT, Santos EM et al. Cuidados com o uso de coeficiente de determinação na análise do ajuste de modelos volumétricos. In: Anais do $4^{\circ}$ Simpósio Latino-americano sobre Manejo Florestal, 2008, Santa Maria. Santa Maria: UFSM; 2008.

Soares CPB, Paula F No, Souza AL. Dendrometria e inventário florestal. Viçosa: Editora UFV; 2006. 276 p. 